\title{
Emploi d'une suceuse hydraulique transformée pour les prélèvements quantitatifs dans les substrats meubles infralittoraux
}

\author{
Henri Massé \\ Station Marine d'Endoume, Marseille, France
}

\begin{abstract}
Use of a transformed hydraulic dredge for quantitative sampling in the loose infralittoral substrate. BRETT (1964) has constructed a new apparatus for sampling macrofauna of the marine benthos. This apparatus is derived from the "gold sucker" employed by ScuBa divers for underwater work. It was used from 1965 for research on the productivity of some sand bottom communities (Venus gallina community of Petersen). Some improvements have been made to obtain a good quantitative sampler. A comparison has been made between the biomasses obtained with an "Orange-Peel" grab and the hydraulic aspirator of BRETT, respectively; advantages and disadvantages are discussed.
\end{abstract}

\section{DESCRIPTION DE L'APPAREIL}

L'appareil que nous utilisons à la Station Marine d'Endoume est dérivé de celui décrit par BRETT (1964). Sa construction est simple et peu onéreuse. Il comprend essentiellement, une moto-pompe d'une puissance de 3,5 C.V. pouvant débiter $15 \mathrm{~m}^{3}$ d'eau à l'heure pour une dénivellation de $30 \mathrm{~m}$. Il s'y ajoute un tuyau d'aspiration, de $5 \mathrm{~m}$ de long et $40 \mathrm{~mm}$ de diamètre, muni d'une crépine et un tuyau de refoulement de 30 à $40 \mathrm{~m}$ de long et $30 \mathrm{~mm}$ de diamètre. Ce tuyau de refoulement est raccordé à un injecteur d'un diamètre de $15 \mathrm{~mm}$ qui amène de l'eau sous pression au centre d'un tuyau rigide en tôle galvanisée de $150 \mathrm{~mm}$ de diamètre. Ce tuyau en tôle est immergé et plein d'eau, le jet sous pression a pour rôle d'entraîner la colonne d'eau contenue dans le tuyau d'après le principe de l'effet Venturi. Une fois la colonne d'eau mise en mouvement il se crée une dépression à l'entrée du tuyau, c'est cette dépression qui est utilisée pour aspirer le sédiment et la faune qui s'y trouve.

Il suffit de fixer un sac en filet de nylon à la sortie de l'eau pour tamiser, sous l'effet de la pression, le sédiment et retenir les animaux. Une vanne placée au niveau de l'injecteur permet de contrôler le débit de l'eau, un système d'attache pratique par galons coulissants permet d'effectuer rapidement les changements des sacs de récolte et de les fermer. Pour obtenir des prélèvements quantitativement comparables, il convient d'utiliser un cylindre métallique muni de deux poignées, sa section est de $0,1 \mathrm{~m}^{2}$ et une génératrice a été graduée de 0 à $40 \mathrm{~cm}$. Je pense que l'emploi d'un tel cylindre 
est préférable à celui du simple cadre utilisé par BRETT (1964). En effet, si le volume à prélever n'est pas bien délimité par les parois rigides du cylindre il se produit au fur et à mesure du prélèvement des affaissements du sédiment qui faussent complètement l'évaluation du volume et de la surface prospectée.

La manipulation en plongée est la suivante: il faut deux plongeurs, ce qui satisfait aux règles de sécurité, l'un se charge du cylindre, l'autre de la suceuse. Le premier enfonce le cylindre dans le sédiment jusqu'à la profondeur désirée par un mouvement de va et vient simultané et opposé sur les poignées, le second présente le tuyau d'aspiration qui a été coudé pour faciliter la succion à l'intérieur du cylindre. Le premier opérateur peut alors contrôler de visu le prélèvement et en diriger le déroulement. En général, dans un sable ou un sable vaseux, il faut compter 2 minutes pour aspirer un contenu de cylindre de $30 \mathrm{dm}^{3}$ et de 30 à 40 minutes pour effectuer un prélèvement de 1 métre carré de surface et $30 \mathrm{~cm}$ de profondeur. Pour ce qui est de la profondeur d'utilisation de cet appareil, compte tenu de la puissance du moteur de la pompe et des temps de prélèvement, il peut être utilisé efficacement jusqu'à 30 mètres. Toutefois, au-delà de 20 métres la durée des paliers de décompression limite vite le nombre des prélèvements possibles. L'auteur utilise depuis plus d'un an ce mode de collecte de la macrofaune dans des fonds de sable fin propre où se rencontrent les peuplements à base de Tellina, Venus, Mactridae, correspondant à la "Venus gallina community" de Petersen; et à la «biocoenose des sables fins bien calibrés» de Peres \& Picard (1964).

\section{AVANTAGES DE LA MÉTHODE}

D'abord sur le plan régional, alors que dans les mers à marées une grande partie des peuplements étudiés découvrent à marée basse, en Méditerranée ils restent toujours immergés. De plus, comme il s'agit de fonds peu profonds, ils sont soumis à l'action parfois violente des houles, aussi, les animaux ont souvent une aptitude particulière à l'enfouissement et échappent à la drague ou aux bennes de type classique. Voici quelques espèces qui ont été obtenues couramment de cette façon alors qu'elles étaient considérées comme rares: un Balanoglosse (sans doute Balanoglossus clavigerus), Echinocardium cordatum et son commensal Montacuta ferruginosa qui dans les petits fonds ( $5 \mathrm{~m}$ de profondeur) vivent en moyenne à 10 à $15 \mathrm{~cm}$ dans le sédiment, la Squille $L y$ siosquilla eusebia qui vit dans un profond terrier, Sipunculus nudus et les Actinies Edwarsidae qui peuvent s'enfoncer rapidement, les Pelecypodes Solecurtus strigillatus, Solemya togata, Pharus legumen, Ensis ensis, Solen marginatus, qui s'enfoncent très rapidement au moindre choc. Enfin, citons le cas particulier de Loripes desmaresti (Payreaudeau) qui vit entre 10 et $15 \mathrm{~cm}$ de profondeur relié à la surface par un tube muceux incrusté de grains de sable et sécrété par son pied modifié, long et vermiforme. Tous ces animaux, qui ne sont pas des espèces rares, constituent autant de pertes, fréquentes à la benne classique, dans l'évaluation correcte de la biomasse. Il faut y ajouter les pertes, très importantes, dues au sectionnement des animaux (en particulier des Polychètes) par les mâchoires des bennes.

Notons en passant que ce mode de prélèvement est particulièrement utile pour procurer du matériel en bon état aux biologistes; ainsi, les Owenia et les Phoronis, 
qui ont des tubes bien ancrés dans le sédiment, peuvent être ramenés entiers pour des études histologiques. Enfin, sur le plan purement quantitatif, les avantages de la suceuse sont d'abord de permettre le contrôle de visu du prélèvement, ce qui constitue

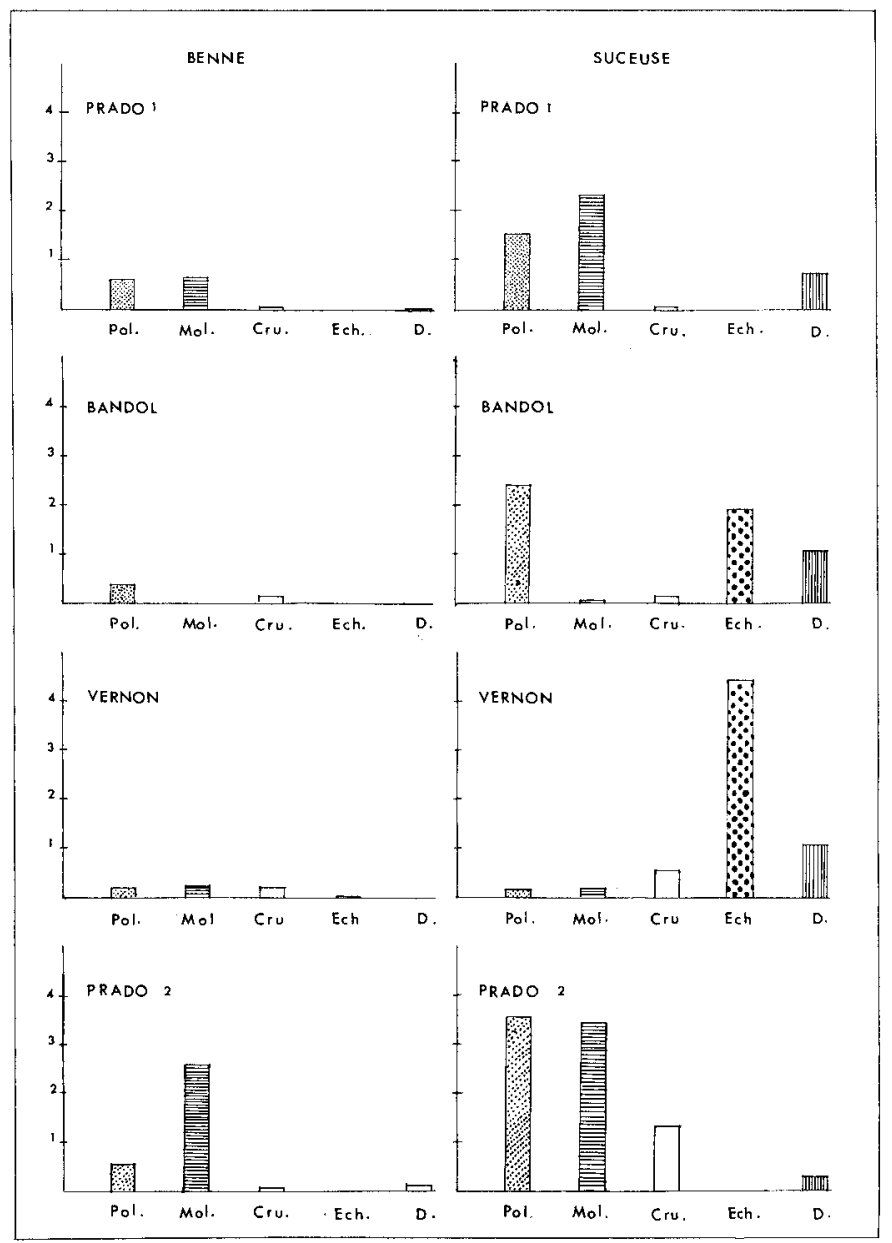

Fig. 1: Les biomasses obtenues en poids secs par la suceuse surpassant les résultats obtenus par la benne à la station de Prado 13,54 fois, à la station de Bandol 11,2 fois, à la station de Vernon 9,3 fois, à la station de Prado 22,85 fois. Ces données prouvent l'avantage de la suceuse sur la benne, en mayenne les biomasses obtenues avec la suceuse sont 6,7 fois plus élevées. Les autres détails se trouvent dans le texte

une garantie totale de régularité et de comparabilité des résultats. L'emploi d'un cylindre délimitant de façon précise la surface et la profondeur du prélèvement constitue d'autre part une garantie indiscutable de l'identité des échantillonnages. Voici, pour illustrer ce propos, une figure regroupant une série de comparaisons effectuées dans différents biotopes à différentes saisons. Dans la partie gauche de la figure 1 on trouve 
les différentes valeurs des biomasses obtenues à partir de prélèvements faits à la benne (du type Orange-peel) et dans la partie de droite les valeurs correspondantes obtenues par des prélèvements simultanés faits à la suceuse. Les différents groupes zoologiques ont été séparés; voici le sens des abbréviations employées: Pol. = Polychètes, Mol. $=$ Mollusques, Cru. $=$ Crustacés, Ech. $=$ Echinodermes, D. $=$ divers ou varia. Les biomasses sont exprimées en gramme par métre carré et il s'agit de poids sec d'animaux décalcifiés; de plus, les estomacs des limivores ont été vidés de leur contenu. Voici les détails des 4 stations:

Station Prado 1: La biomasse des Polychètes est plus importante à la suceuse. Pour les Mollusques, la différence de poids est due à la présence de Loripes desmaresti espèce trop profondement enfouie pour être prise à la benne. Sous la rubrique «divers», la suceuse a ramené deux Actinies du genre Edwarsia, 30 fois plus de Phoronis psammophila et un jeune Cérianthe. Ce qui donne une biomasse totale de $1,3 \mathrm{~g} /$ métre carré à la benne contre, $4,6 \mathrm{~g} / \mathrm{m}^{2}$ à la suceuse.

$\mathrm{S}$ tatio n B a ndol: Là encore, la suceuse ramène plus de Polychètes, en particulier de grosses Clymene et des Aricia. Pour les Echinodermes, la suceuse à ramené un Echinocardium cordatum trop profondément enfoui pour la benne. Enfin dans la colonne "divers» un Sipunculus nudus et une Edwarsia expliquent l'avantage de la biomasse du prélèvement fait à la suceuse. La biomasse totale à la benne est de $0,5 \mathrm{~g} / \mathrm{m}^{2}$ et de $5,7 \mathrm{~g} / \mathrm{m}^{2}$ à la suceuse.

$\mathrm{Station} V$ ernon: Ce biotope qui est particulièrement pauvre renferme toutefois en abondance l'espèce Echinocardium cordatum qui vit enfouie au-delà de $10 \mathrm{~cm}$ ce qui explique la différence des valeurs obtenues à la benne et à la suceuse. A la rubrique «divers» une grosse Nemerte est responsable de la différence de poids. La biomasse totale à la benne est de $0,7 \mathrm{~g} / \mathrm{m}^{2}$ et de $6,5 \mathrm{~g} / \mathrm{m}^{2}$ à la suceuse.

Station Prado 2: Ce sont une fois de plus les Polychètes qui ont fait pencher la balance du côté de la suceuse. La biomasse totale est de $3,4 \mathrm{~g} / \mathrm{m}^{2}$ pour la benne et de $8,7 \mathrm{~g} / \mathrm{m}^{2}$ pour la suceuse.

\section{INCONVENIENTS DE LA SUCEUSE}

Un des inconvénients de ce procédé réside dans le fait qu'il exige d'être pratiqué en plongée ce qui limite son emploi et sa généralisation, donc la possibilité de faire des comparaisons valables entre les biomasses des différentes mers. Son emploi reste difficile à une grande échelle, car c'est un travail pénible surtout dans les eaux froides et les eaux troubles. C'est d'ailleurs pour remédier à ces inconvenients qu'un modèle entièrement autonome de suceuse, basé sur le même principe, est en cours de réalisation et d'expérimentation (Reys, 'True \& True-Schlenz 1966).

On peut aussi objecter que le fait d'enfoncer le cylindre avant le prélèvement provoque un ébranlement et le retrait en profondeur des formes fouisseuses; toutefois, la possibilité de prélever sur $30 \mathrm{~cm}$ et le fait de contrôler le déroulement du prélèvement de visu permettent de remédier à ce défaut. 


\section{CONCLUSION}

En conclusion, je pense que ce mode de prélèvement de la macrofaune est extrêmement intéressant, tant au point de vue quantitatif que qualitatif. Au point de vue quantitatif, les valeurs données ici sont suffisamment éloquentes, de plus, le contrôle de visu du prélèvement et l'emploi du cylindre de référence mettent la méthode à l'abri de toutes les critiques formulées à l'égard des bennes, à savoir l'irrégularité des surfaces et volumes prélevés.

Sur le plan qualitatif, la prospection peut donner lieu à la recherche systématique de la nature des traces et des terriers non identifiés. Enfin l'observation du prélèvement, de son déroulement, peut fournir de précieuses indications d'ordre écologique, telles la position et la statigraphie des animaux dans leur biotope.

\section{RESUME}

1. Cette communication a pour but de montrer l'avantage de la «suceuse hydraulique» de BRETT (1964) par rapport à une benne de type classique.

2. Voici les résultats globaux obtenus pour 4 stations différentes prospectées simultanement à la benne et à la «suceuse hydraulique». (a) Station Prado 1: Biomasse à la benne «Orange-Peel» $1,3 \mathrm{~g} / \mathrm{m}^{2}$, à la «suceuse hydraulique» $4,6 \mathrm{~g} / \mathrm{m}^{2}$. (b) Station Bandol: Biomasse à la benne "Orange-Peel» $0,5 \mathrm{~g} / \mathrm{m}^{2}$, ̀̀ la «suceuse hydraulique» $5,7 \mathrm{~g} / \mathrm{m}^{2}$. (c) Station Vernon: Biomasse à la benne «Orange-Peel» $0,7 \mathrm{~g} / \mathrm{m}^{2}$, à la «suceuse hydraulique» $6,5 \mathrm{~g} / \mathrm{m}^{2}$. (d) Station Prado 2: Biomasse à la benne «OrangePeel» $3,4 \mathrm{~g} / \mathrm{m}^{2}$, à la «suceuse hydraulique» $8,7 \mathrm{~g} / \mathrm{m}^{2}$. Les biomasses sont exprimées en poids sec d'animaux décalcifiés en gramme par métre carré.

\section{LITTÉRATURE CITÉE}

BRETT, C. E., 1964. A portable hydraulic diver-operated dredge - sieve for sampling subtidal macrofauna. J. mar. Res. 22, 205-222.

REYS, J. P., 1963. Les prélèvements quantitatifs du benthos de substrats meubles. Terre Vie 1.

- True, M. A. \& True-Schlenz, R., 1966. Un nouvel appareil de prélèvement quantitatif de substrats meubles. Int. oceanogr. Congr. 2, 350.

Peres, J. M. \& Picard, J., 1964. Nouveau manuel de bionomie benthique de la Mer Méditerranée. Ed. revue et augm. Recl Trav. Stn mar. Endoume 31 (47), 1-137.

\section{Discussion suivant le rapport de Massé}

BARNETT: I am very interested to hear that M. MASSÉ has found such big differences between the grab and his suction sampler. At Millport we too have been able to show big differences between sand samples taken with a shallow digging vAN VEEN grab and the deeper coring KNUDSEN sampler. I think that M. Massé must be working with quite a loose sediment. At Millport we built a suction sampler following BRETr's design, and while it worked quite well in a Venus gallina sand, it was very slow on a coarser hard packed sand containing quite a lot of silt and stones. On these sediments we found that the airlift was very successful. It is diffi- 
cult to compare two different suction samplers being used on two different sediments, but I would like to ask if you have any data on the suction pressures produced at the mouth of your sampler?

Massé: Non, je n'ai pas mesuré cette pression, mais je peux vous dire que cet appareil peut aspirer des galets gros comme mon poing. Le rapport des diamètres de l'injecteur et du tuyau de la suceuse, ainsi que le rapport des longueurs des différentes parties de l'appareil doivent faire l'objet d'expérimentations pour obtenir un rendement maximum. Je pense que la suceuse de Bret'T doit pouvoir fonctionner dans votre type de fond si le débit de votre pompe est suffisant. Le seul problème à mon avis est d'enfoncer à la main le cylindre de référence, jusqu'à $60 \mathrm{~cm}$, car votre sédiment est très compact et riche en gros débris. Toutefois, dans la plupart des prélèvements benthiques il n'est pas utile de prélever jusqu'à $60 \mathrm{~cm}$ de profondeur.

BARNETT: Nevertheless, I think it would be good to measure the suction pressures produced by both the BRETT sampler and the airlift suction pipe. It is interesting that in your sands you are able to sink in your cylinder by hand. We can do the same in Venus sands in Scotland but not in the coarser sediments we are working with. When we tried to do this, we found that the vibrations produced by the semi-rotary action of the cylinder make the sand fluid within the cylinder, and some animals float to the surface of the sand. If you are interested in the vertical distribution of organisms in the sediment, is this not a disadvantage?

Massé: Je n'ai pas observé ces phénomènes de tixotropie dans le sable propre où je travaille et même s'il est un peu vaseux. Pour ce qui est de l'observation de la distribution verticale des animaux, il est certain que la simple présence des plongeurs peut faire fuir en profondeur certaines espèces qui vivent normalement à la surface du sédiment. Seule la connaissance de l'éthologie de ces espèces permet de remédier à cet inconvénient.

BARNETT: Finally, do I understand that when you have emptied the sand from your cylinder, you are able to pull it out by hand: When we are sampling to a depth of $60 \mathrm{~cm}$ in the sand, three men are unable to pull out the cylinder. This is why we have to use compressed air. Have you had similar problems?

Massé: Dans les sédiments où j'ai pu essayer l'appareil de BRETr j'ai toujours extrait le cylindre assez facilemcnt, il est vrai que je ne l'enfonce qu'à 30 ou $40 \mathrm{~cm}$ de profondeur et non à $60 \mathrm{~cm}$ comme vous le faites.

JoNEs: Conditions are not really too bad in northern waters. I have been looking for such an apparatus to collect longer burrowing and tube-living animals. Can you tell me about the availability and approximate cost?

Massé: Je crois avoir dit au début de cet exposé que l'acquisition de cet appareil est peu onéreuse, vous pouvez le réaliser vous-même. Dans cette publication je donne les caractéristiques de l'appareil que j'utilise et BRETT (J.mar. Res. 22, 1964) a décrit son appareil. Je ne pense pas qu'un appareil de ce type soit commercialisé.

Gamulin-Brida: Je pense que la «suceuse hydraulique» représente une contribution très utile pour la technique de prélèvements; est-ce qu'il est possible de l'employer dans les profondeurs plus grandes, par exemple 100 mètres, et sans le contrôle des plongeurs?

Massé: J'ai dit dans mon exposé que pour faire des prélèvements plus profonds là où les plongeurs ne peuvent pas aller il faut un appareil autonome. Cet appareil, dont il existe un prototype (Reys, True \& True-Schlenz; 2nd Int. oceanogr. Congr. Moscow 1966, Abstr., 350) est actuellement à l'étude et en cours de perfectionnement à la Station Marine d'Endoume, Marseille. 